OPEN ACCESS

Edited by:

Jian Zhong,

Shanghai Ocean University, China

Reviewed by:

Barbara Sanavio,

Fondazione IRCCS Istituto

Neurologico Carlo Besta, Italy

Silvia Castro Coelho,

Faculdade de Engenharia da

Universidade do Porto, Portugal

*Correspondence:

Steven Lenhert

lenhert@bio.fsu.edu

Specialty section:

This article was submitted to

Nanobiotechnology,

a section of the journal

Frontiers in Materials

Received: 09 June 2016 Accepted: 02 December 2016

Published: 23 December 2016

Citation:

Ghazanfari L and Lenhert S (2016) Screening of Lipid Composition for Scalable Fabrication of Solvent-Free

Lipid Microarrays.

Front. Mater. 3:55

doi: 10.3389/fmats.2016.00055

\section{Screening of Lipid Composition for Scalable Fabrication of Solvent-Free Lipid Microarrays}

\author{
Lida Ghazanfari and Steven Lenhert*
}

Department of Biological Sciences, Integrative NanoScience Institute, Florida State University, Tallahassee, FL, USA

Liquid microdroplet arrays on surfaces are a promising approach to the miniaturization of laboratory processes such as high-throughput screening. The fluid nature of these droplets poses unique challenges and opportunities in their fabrication and application, particularly for the scalable integration of multiple materials over large areas and immersion into cell culture solution. Here, we use pin spotting and nanointaglio printing to screen a library of lipids and their mixtures for their compatibility with these fabrication processes, as well as stability upon immersion into aqueous solution. More than 200 combinations of natural and synthetic oils composed of fatty acids, triglycerides, and hydrocarbons were tested for their pin-spotting and nanointaglio print quality and their ability to contain the fluorescent compound tetramethylrhodamine B isothiocyanate (TRITC) upon immersion in water. A combination of castor oil and hexanoic acid at the ratio of 1:1 (w/w) was found optimal for producing reproducible patterns that are stable upon immersion into water. This method is capable of large-scale nanomaterials integration.

Keywords: high-throughput screening, droplet microarray, lipid, lipophilic drug, nanointaglio

\section{INTRODUCTION}

A fundamental goal of nanotechnology is to integrate top-down nanofabrication processes with bottom-up chemical assembly to reliably fabricate larger, more complex devices with molecular scale components (Rohrer, 1996). Liquid microdroplet arrays on surfaces are a promising approach toward achieving this goal by allowing multiple solutions to be integrated on a chip (Gosalia and Diamond, 2003; Popova et al., 2016). In principle, each droplet can be viewed as a microscopic test tube, allowing a density of containers limited only by droplet size and the ability to place different reagents into each droplet. For instance, an array with one droplet per square micrometer would allow 100 million containers on $1 \mathrm{~cm}^{2}$ surface. The potential in highthroughput screening (HTS), with the state of the art being 10-30 wells $/ \mathrm{cm}$, is comparable to the difference in capabilities between early vacuum tube-based computer mainframes and today's solid-state computers.

Modern HTS requires robotics, liquid-handling devices, sensitive detectors, and software for data processing and control in order to perform millions of pharmacological tests on samples in parallel. Current robotic systems are burdened by several issues, such as high costs, poor reliability of data, standardization of data types, rapid and accurate dispensing of very small liquid volumes, and uncontrolled evaporation of dispensed liquids from Comley (2006). One promising approach to miniaturization of HTS is microfluidics. Microfluidic systems enable serial processing and analysis and, furthermore, can accomplish massive parallelization through 
efficient miniaturization and multiplexing (Hong et al., 2009). In particular, droplet microfluidics use small droplets, typically water suspended in oil, to confine reagents and/or cells (Anna et al., 2003; Kim et al., 2015). A challenge in this field is that the droplets move and mix in solution, and a chemical tracker is therefore typically included in the drop for identification. Droplet microarrays provide a different solution to this technical challenge by attaching the droplet to a surface, so that its composition is known by its position in the array, at the cost of limiting the array to two dimensions (Gosalia and Diamond, 2003; Mugherli et al., 2009; Arrabito et al., 2013; Sun et al., 2015; Popova et al., 2016).

Microarrays of covalently attached monolayers are well established and allow the simultaneous analysis of thousands of chemical entities within a single experimental step (Cahill, 2001; Heller, 2002; Pirrung, 2002; Howbrook et al., 2003; Hook et al., 2006; Ma and Horiuchi, 2006). Biomolecules commonly immobilized on microarrays include proteins (Cahill, 2001), oligonucleotides (Heller, 2002; Pirrung, 2002; Howbrook et al., 2003), polymerase chain reaction products (Heller, 2002; Pirrung, 2002), peptides (Cahill, 2001; Howbrooketal., 2003), lipids (Howbrooketal., 2003; Hook et al., 2006), and carbohydrates (Ma and Horiuchi, 2006). Covalent small molecule microarrays are useful for screening for interactions with the surfaces of adherent cells. However, targets inside of the cell are inaccessible to this approach. Alternatives include embedding the small molecules into a matrix such as a hydrogel and allowing them to diffuse out (Bailey et al., 2004), a sandwich assay composed of microwells that are addressable by individual posts (Wu et al., 2011), or by generating arrays of microscopic water droplets for cell culture (Popova et al., 2016). These methods are promising for water-soluble compounds. However, an estimated $40 \%$ of approved drugs in the market and nearly $90 \%$ of molecules in the developmental pipeline are poorly water soluble (Kalepu and Nekkanti, 2015). This poses a challenge for delivery to cells through aqueous solution. We use lipid multilayer (or droplet) microarrays to temporarily immobilize lipophilic compounds onto a surface, allowing cellular uptake and quantitative dose-response curves (Kusi-Appiah et al., 2012; Kusi-Appiah et al., 2015). A crucial property of lipid multilayer microarrays for drug screening applications is that the layer must be thicker than a single monolayer or bilayer in order to contain enough drug to reach biologically relevant dosages upon cellular uptake.

Lipid multilayer microarrays have been be fabricated by dip pen nanolithography (Lenhert et al., 2007), polymer pen lithography (Hirtz et al., 2015), nanointaglio printing (Lowry et al., 2014), and evaporative edge lithography (Vafai et al., 2015). Here, we use nanointaglio printing, which is a printing mode where ink is transferred from the recesses of a stamp, allowing for control of lipid multilayer film thicknesses by the stamp dimensions as well as the amount of ink on the stamp (Nafday et al., 2012). We have previously demonstrated that three different lipids can be integrated over larger areas by pin spotting of lipid solutions onto a palette, which is subsequently used to ink the intaglio stamp (Lowry et al., 2014). In order to scale this process up for integration of thousands of different lipid encapsulated drug candidates for HTS, several obstacles must be overcome. First of all, we have previously used liposomal solutions in water for the microarray process, yet solvent evaporation becomes an issue as more compounds are added. Second, immersion of the lipid microarrays into water poses a challenge, as the lipids can sometimes be swept away upon addition of aqueous solution. In order to solve these problems, we here screen different fluid lipid carriers as a suitable matrix for solvent-free microarraying followed by intaglio printing and immersion into water. Our main objective here is to identify a fluid lipid composition capable of containing lipophilic small molecules and compatible with pin spotting and microarraying so that this process can be scaled up for HTS applications (Figure 1).

\section{MATERIALS AND METHODS}

\section{Components}

As shown in Figure 2, the components of the lipid formulations screened here include fatty acids [octanoic (caprilic) acid, hexanoic (caproic) acid, oleic acid, linoleic acid], triglycerols (olive oil, soybean oil, sesame oil, peanut oil, linseed oil, corn oil, cottonseed oil, castor oil, lavender oil, mineral oil, sunflower oil, safflower oil, canola oil, fish oil)/hydrocarbon (hexadecane), glycerol,

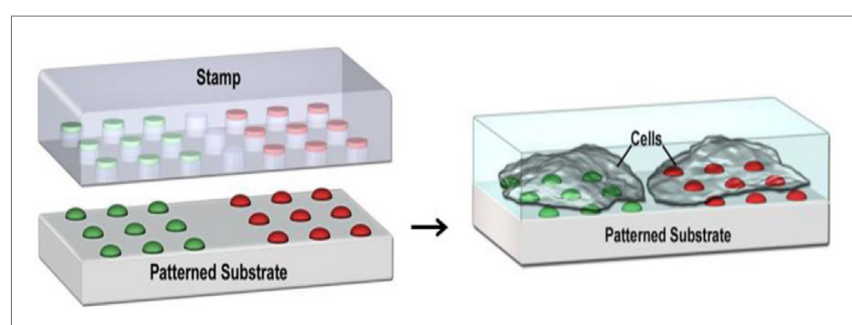

FIGURE 1 | Schematic showing the nanointaglio fabrication process (left) and its application in cell-based high-throughput screening

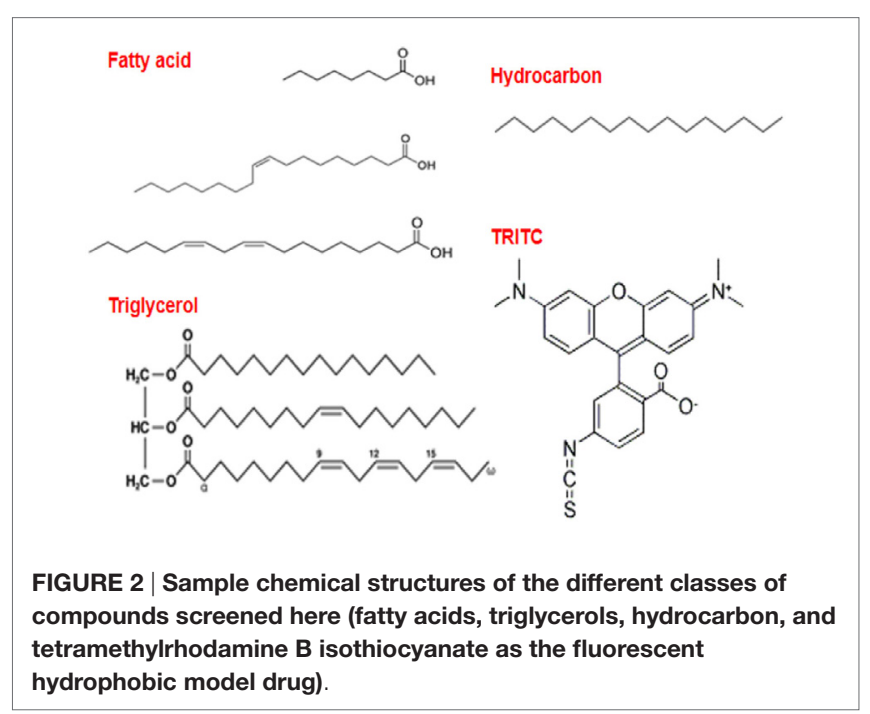


TABLE 1 | List of components [the combinations have the ratio of 1:1 (w/w)].

\begin{tabular}{|c|c|c|c|c|c|c|c|c|}
\hline Hexanoic acid only & 43 & Octanoic and sunflower & 85 & Peanut and cottonseed & 127 & Olive and hexadecane & 169 & Safflower and hexadecane \\
\hline Hexanoic and octanoic & 44 & Octanoic and canola & 86 & Peanut and linseed & 128 & Olive and glycerol & 170 & Safflower and glycerol \\
\hline Hexanoic and oleic & 45 & Octanoic and sesame & 87 & Peanut and safflower & 129 & Soybean oil only & 171 & Sunflower oil only \\
\hline Hexanoic and linoleic & 46 & Octanoic and castor & 88 & Peanut and sunflower & 130 & Soybean and olive & 172 & Sunflower and canola \\
\hline Hexanoic and soybean & 47 & Octanoic and fish & 89 & Peanut and canola & 131 & Soybean and peanut & 173 & Sunflower and sesame \\
\hline Hexanoic and olive & 48 & Octanoic and mineral & 90 & Peanut and sesame & 132 & Soybean and corn & 174 & Sunflower and castor \\
\hline Hexanoic and peanut & 49 & Octanoic and lavender & 91 & Peanut and castor & 133 & Soybean and sunflower & 175 & Sunflower and hexadecane \\
\hline Hexanoic and corn & 50 & Octanoic and hexadecane & 92 & Peanut and fish & 134 & Soybean and cottonseed & 176 & Sunflower and fish \\
\hline Hexanoic and cottonseed & 51 & Octanoic and glycerol & 93 & Peanut and mineral & 135 & Soybean and linseed & 177 & Sunflower and mineral \\
\hline Hexanoic and linseed & 52 & Corn oil only & 94 & Peanut and lavender & 136 & Soybean and safflower & 178 & Sunflower and lavender \\
\hline Hexanoic and safflower & 53 & Corn and cottonseed & 95 & Peanut and hexadecane & 137 & Soybean and canola & 179 & Sunflower and glycerol \\
\hline Hexanoic and sunflower & 54 & Corn and linseed & 96 & Peanut and glycerol & 138 & Soybean and sesame & 180 & Canola oil only \\
\hline Hexanoic and canola & 55 & Corn and safflower & 97 & Linoleic acid only & 139 & Soybean and castor & 181 & Canola and sesame \\
\hline Hexanoic and sesame & 56 & Corn and sunflower & 98 & Linoleic and soybean & 140 & Soybean and fish & 182 & Canola and castor \\
\hline Hexanoic and castor & 57 & Corn and canola & 99 & Linoleic and olive & 141 & Soybean and glycerol & 183 & Canola and hexadecane \\
\hline Hexanoic and fish & 58 & Corn and sesame & 100 & Linoleic and peanut & 142 & Soybean and mineral & 184 & Canola and fish \\
\hline Hexanoic and mineral & 59 & Corn and castor & 101 & Linoleic and corn & 143 & Soybean and lavender & 185 & Canola and mineral \\
\hline Hexanoic and lavender & 60 & Corn and fish & 102 & Linoleic and cottonseed & 144 & Soybean and hexadecane & 186 & Canola and lavender \\
\hline Hexanoic and hexadecane & 61 & Corn and mineral & 103 & Linoleic and linseed & 145 & Fish oil only & 187 & Canola and glycerol \\
\hline Hexanoic and glycerol & 62 & Corn and lavender & 104 & Linoleic and safflower & 146 & Fish and mineral & 188 & Mineral oil only \\
\hline Cottonseed oil only & 63 & Corn and hexadecane & 105 & Linoleic and sunflower & 147 & Fish and lavender & 189 & Mineral and lavender \\
\hline Cottonseed and linseed & 64 & Corn and glycerol & 106 & Linoleic and canola & 148 & Fish and hexadecane & 190 & Mineral and hexadecane \\
\hline Cottonseed and safflower & 65 & Oleic acid only & 107 & Linoleic and sesame & 149 & Fish and glycerol & 191 & Mineral and glycerol \\
\hline Cottonseed and sunflower & 66 & Oleic and linoleic & 108 & Linoleic and castor & 150 & Linseed oil only & 192 & Sesame oil only \\
\hline Cottonseed and canola & 67 & Oleic and soybean & 109 & Linoleic and fish & 151 & Linseed and safflower & 193 & Sesame and castor \\
\hline Cottonseed and sesame & 68 & Oleic and olive & 110 & Linoleic and mineral & 152 & Linseed and sunflower & 194 & Sesame and fish \\
\hline Cottonseed and castor & 69 & Oleic and peanut & 111 & Linoleic and lavender & 153 & Linseed and canola & 195 & Sesame and mineral \\
\hline Cottonseed and fish & 70 & Oleic and corn & 112 & Linoleic and hexadecane & 154 & Linseed and mineral & 196 & Castor oil only \\
\hline Cottonseed and mineral & 71 & Oleic and cottonseed & 113 & Linoleic and glycerol & 155 & Linseed and sesame & 197 & Sesame and Lavender \\
\hline Cottonseed and lavender & 72 & Oleic and linseed & 114 & Olive oil only & 156 & Linseed and castor & 198 & Sesame and hexadecane \\
\hline Cottonseed and hexadecane & 73 & Oleic and safflower & 115 & Olive and peanut & 157 & Linseed and fish & 199 & Sesame and glycerol \\
\hline Cottonseed and glycerol & 74 & Oleic and sunflower & 116 & Olive and corn & 158 & Linseed and lavender & 200 & Castor and fish \\
\hline Octanoic acid only & 75 & Oleic and canola & 117 & Olive and cottonseed & 159 & Linseed and hexadecane & 201 & Castor and mineral \\
\hline Octanoic and oleic & 76 & Oleic and sesame & 118 & Olive and linseed & 160 & Linseed and glycerol & 202 & Castor and lavender \\
\hline Octanoic and linoleic & 77 & Oleic and castor & 119 & Olive and safflower & 161 & Safflower oil only & 203 & Castor and hexadecane \\
\hline Octanoic and soybean & 78 & Oleic and fish & 120 & Olive and sunflower & 162 & Safflower and castor & 204 & Lavender and glycerol \\
\hline Octanoic and olive & 79 & Oleic and mineral & 121 & Olive and canola & 163 & Safflower and sunflower & 205 & Castor and glycerol \\
\hline Octanoic and peanut & 80 & Oleic and lavender & 122 & Olive and sesame & 164 & Safflower and canola & 206 & Lavender oil only \\
\hline Octanoic and corn & 81 & Oleic and hexadecane & 123 & Olive and castor & 165 & Safflower and sesame & 207 & Lavender and hexadecane \\
\hline Octanoic and cottonseed & 82 & Oleic and glycerol & 124 & Olive and fish & 166 & Safflower and fish & 208 & Hexadecane only \\
\hline Octanoic and linseed & 83 & Peanut oil only & 125 & Olive and mineral & 167 & Safflower and mineral & 209 & Hexadecane and glycerol \\
\hline Octanoic and safflower & 84 & Peanut and corn & 126 & Olive and lavender & 168 & Safflower and lavender & 210 & Glycerol only \\
\hline
\end{tabular}

and tetramethylrhodamine B isothiocyanate (TRITC), as the fluorescent hydrophobic model drug, which are purchased from Sigma-Aldrich. The combinations of 1:1 (w/w) liquid lipids and the pure lipids are tested (Table 1). The oil phase must be of high purity and free of undesirable components such as peroxides, pigments, decomposition products, and unsaponifiable matter such as sterols and polymers. Oxidation of oil and drug during preparation and storage must be minimized by manufacturing under a nitrogen atmosphere, as reported by Floyd (1999).

\section{PDMS Stamps}

PDMS micro-well stamps are prepared from a thermoplastic master (EV Group, Inc., Tempe, AZ, USA) cured from a patterned silicon wafer with $5 \mu \mathrm{m}$ diameter wells, $2.5 \mu \mathrm{m}$ deep and $10 \mu \mathrm{m}$ in pitch, covering $19 \%$ of the stamp surface. The silicon wafers are initially cleaned with piranha solution or plasma treated and later passivated with a $0.2 \%$ (by volume) octadecyltrichlorosilane solution in toluene. The PDMS stamp of desired dimensions is prepared from a Sylgard 184 (Dow Corning, Midland, MI, USA) elastomer gel at a ratio of 1:10 curing agent to base prepolymer poured over the thermoplastic master and cured in an oven at $65^{\circ} \mathrm{C}$ overnight.

\section{Ink Preparation}

For integration of multiple inks, TRITC, as a model drug, is added to the liquid lipids at a proportion of $1 \%$ by mass for arraying, screening, and microscopy. The results are microarrayed in an array pattern onto a PDMS ink palette.

\section{Microarraying Lipid Components}

The different lipid solutions are microarrayed from standard 384-well microtiter plates (Axygen, Inc., PMI110-07 V1, Union 
City, CA, USA) using a Microarrayer (Arrayit Corporation, ARYC) onto the PDMS palettes (Figure 3 and Figure S1 in Supplementary Material), using a $200 \mu \mathrm{m} 4 \times 4$ stainless steel microspot pin tool. Microarray pins are washed to ensure no cross-contamination between inks. It is found that 2 min washes in acetone and then water, followed by $30 \mathrm{sec}$ of drying sufficed.

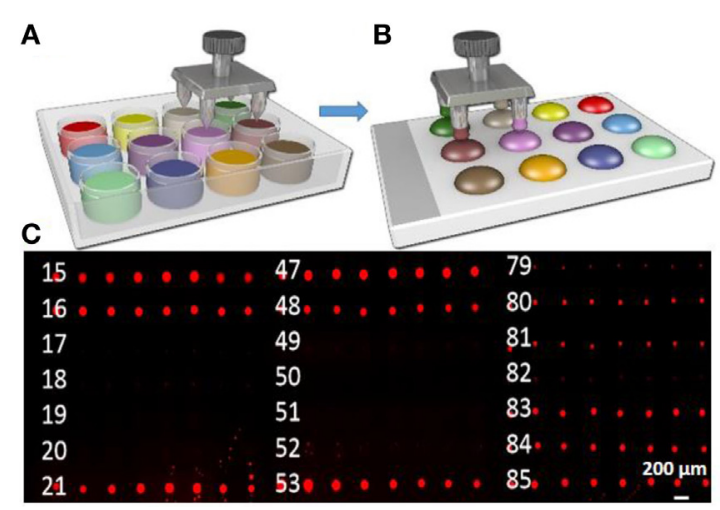

FIGURE 3 | Pin spotting screening of liquid lipid-based components (A,B) Schematic illustrating the process of inking of lipid spots; (C) fluorescence micrographs of palette. Scale bar is $200 \mu \mathrm{m}$.

\section{Intaglio Printing}

For lipid/dye combination stamping on the cover glass palette surfaces, the PDMS stamp is inked and placed in contact with the substrate. A structured PDMS stamp is inked by pressing the patterned surface onto the ink palette (Lowry et al., 2014). The stamping procedure combines the topographical control of nanoimprint lithography and throughput of microcontact printing with the scalability of pin spotting. The stamps are left in direct contact with the surface and uniform, firm pressure (about $45 \mathrm{~N}$

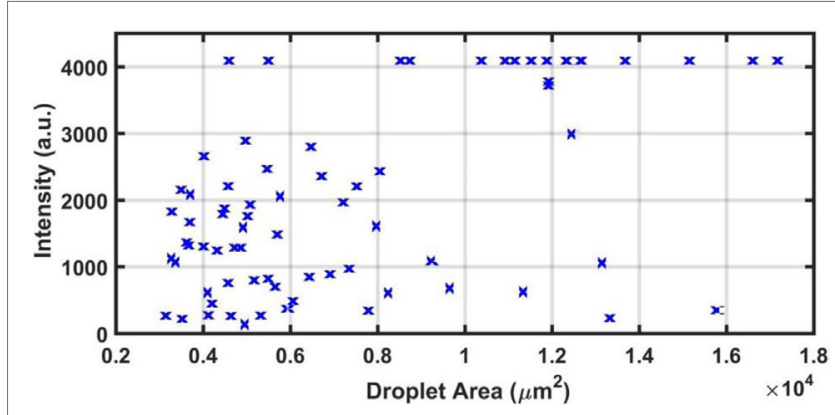

FIGURE 5 | Plot of intensity versus droplet area of pin spotting screening of liquid lipid-based components. The brightest dots are saturated in fluorescence intensity, indicating sufficient dye content for our purposes.

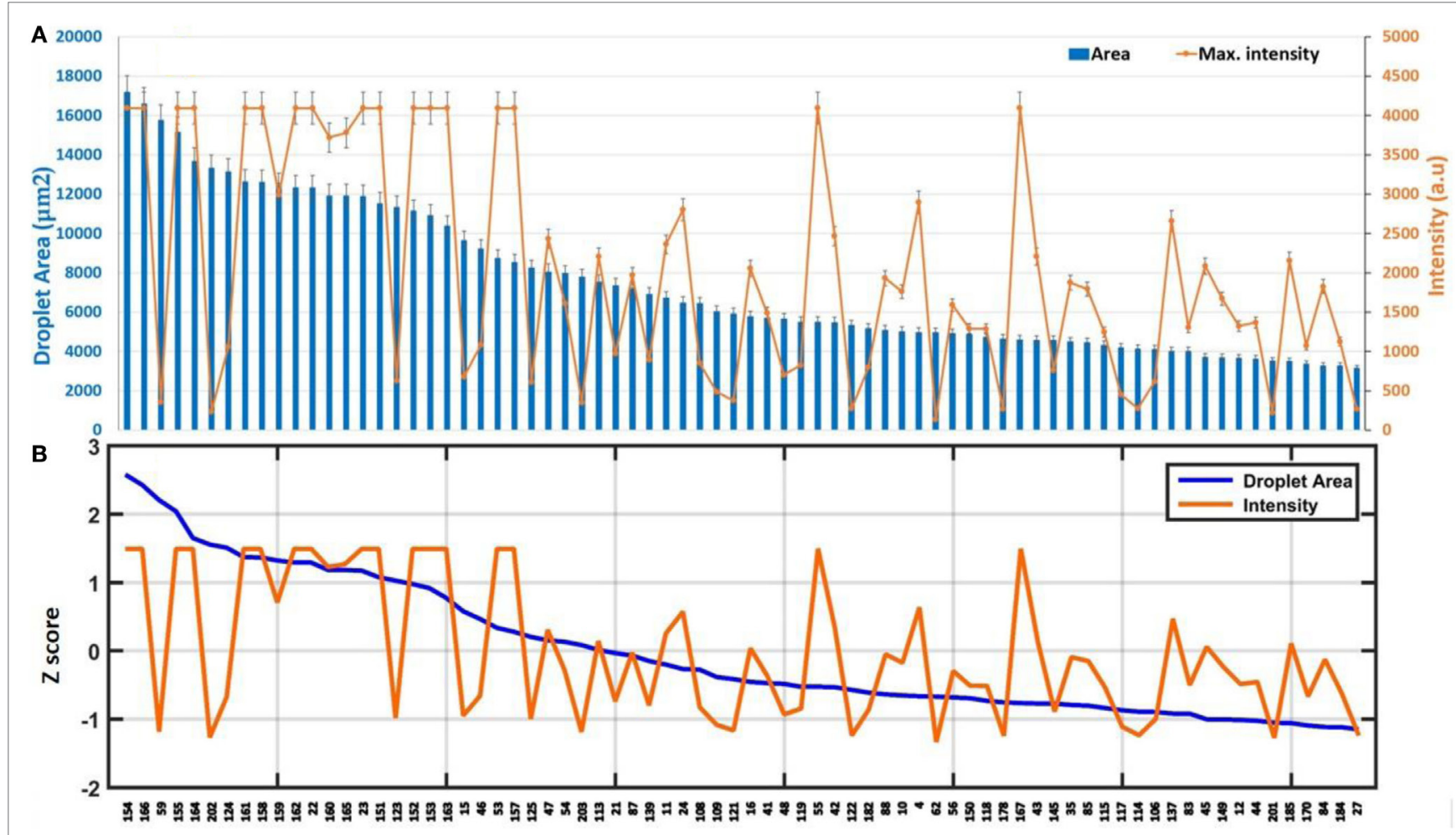

FIGURE 4 | (A) Quantitative analysis of pin spotting screening of liquid lipid-based components in terms of droplet area and intensity. Error bars represent the SEM of at least nine different spots. (B) $Z$ value of the components. 
as measured on a bathroom scale) is applied for $\sim 10 \mathrm{sec}$ before careful removal and printing the next pattern. Excess material is removed by sacrificially printing four to six times before pattern

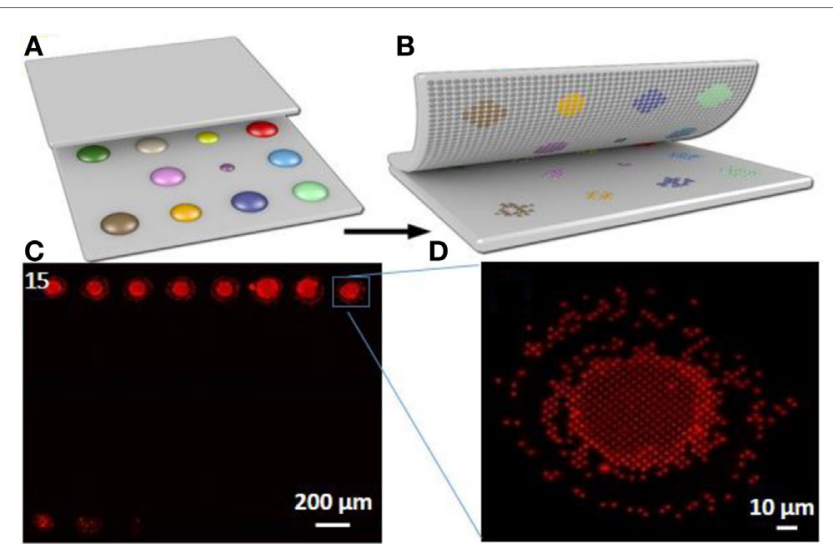

FIGURE 6 | Nanointaglio print compatibility screening of liquid lipid-based components in terms of area and intensity. $(A, B)$

Schematic illustrating the process of nanointaglio printing of lipid spots; (C) fluorescence micrograph of a lipid microarray printed using the nanointaglio method; (D) magnified section of (C) indicated by blue square in (C). would print uniformly. Image analysis for area and intensity of the droplets is done by NIH ImageJ software (http://rsb.info.nih. gov/ij/) (Figure 4A; Figure S2 in Supplementary Material).

Quantitative analysis of pin spotting screening of liquid lipid-based components, together with the $Z$ value of the components, is shown in Figure 4. Furthermore, a scatter plot of the two parameters tested (intensity and droplet area) is provided (Figure 5).

For Figure 6 and Figure S3 in Supplementary Material, nanointaglio patterns are printed on glass coverslip substrates. Furthermore, quantitative analysis of the printing compatibility screening of liquid lipid-based components and their $Z$ values are shown in Figure 7. The description of the correlation of intensity and print area is provided in Figure 8.

\section{Lipid Nanopattern Storage and Immersion}

After nanointaglio fabrication, lipid patterns are stored in a nitrogen glovebox (Mbraun, Inc., Model Labstar (1200/780), Stratham, $\mathrm{NH}$, USA) to prevent them from possible oxidation. The nitrogen environment stabilizes the lipid nanostructures by dehydration prior to immersion in water (Lenhert et al., 2010). Then Millipore water is applied for $1 \mathrm{~h}$, using a syringe directly over a section of the lipid pattern on a microscope stage while the pattern is being imaged on fluorescence microscope (Figure 9). Moreover,

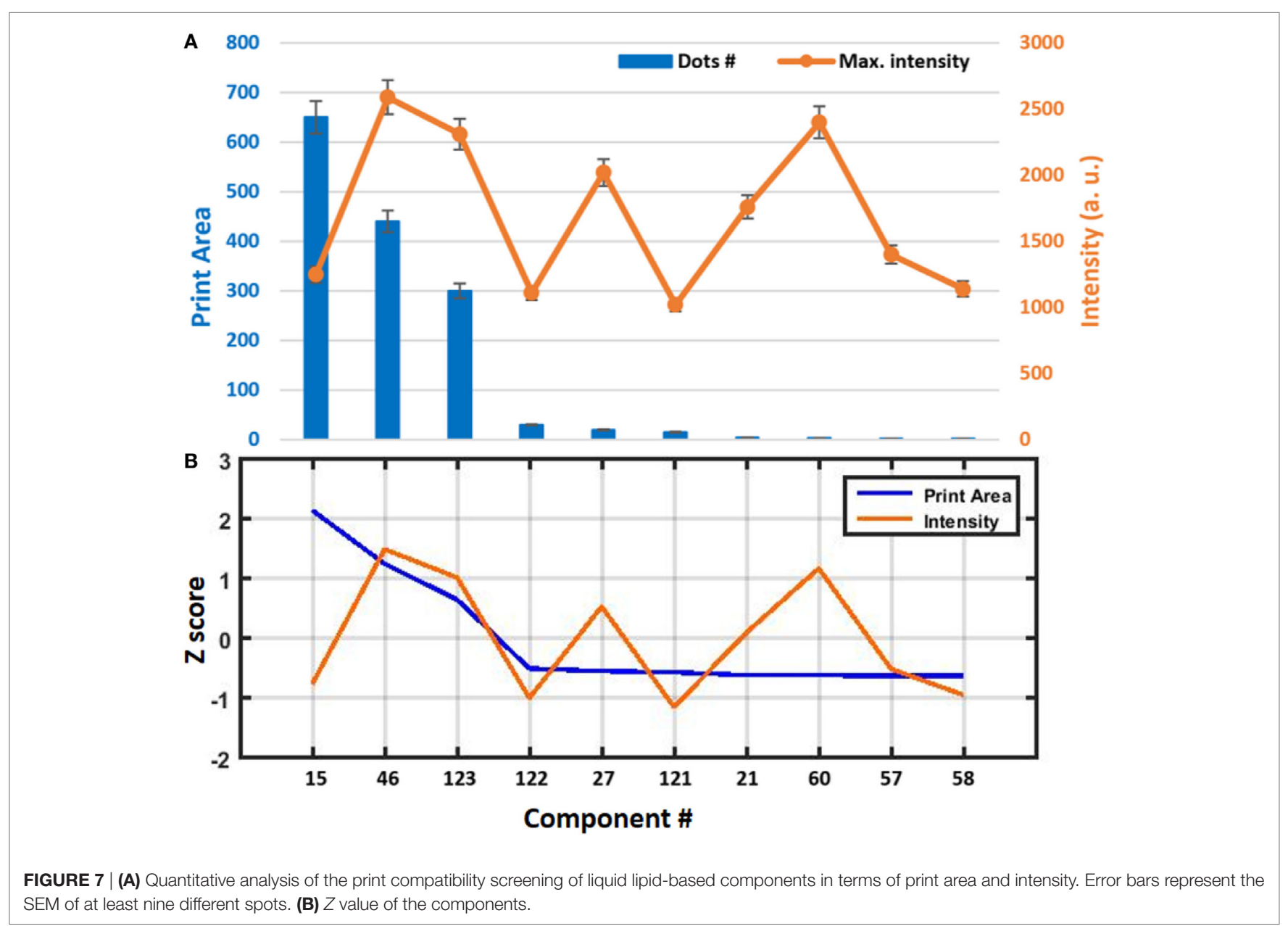




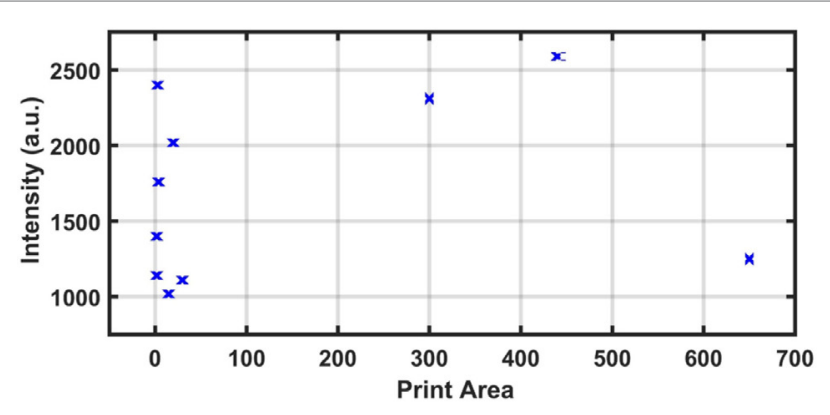

FIGURE 8 | Plot of intensity versus print area of printing compatibility screening of liquid lipid-based components

we repeat the same experiment for the selected components over a large pattern. This time after being imaged for $1 \mathrm{~h}$, the patterns are kept at ambient temperature $\left(25^{\circ} \mathrm{C} \pm 2 \%\right)$ for $72 \mathrm{~h}$ and are imaged again by fluorescence microscopy.

\section{Preparation of Immersion Chamber}

A $0.5 \mathrm{~cm}$ diameter cork bore is used to create cutouts in PDMS pieces $1 \mathrm{~cm}$ wide by $3 \mathrm{~cm}$ long by $0.5 \mathrm{~cm}$ thick. This chamber is placed on a glass slide with the lipid patterns to create an enclosed space to contain solution for experiments.

\section{Characterization and Imaging Techniques}

A Ti-E epifluorescence inverted microscope (Nikon Instruments, Melville, NY, USA) fitted with a Retiga SRV (QImaging, Canada) CCD camera (1.4 MP, Peltier cooled to $-45^{\circ} \mathrm{C}$ ) is used for fluorescence and bright-field imaging of the lipid patterns on glass surfaces. All experiments are performed at ambient temperature.

\section{Statistical Analysis}

All experiments are performed at least in triplicate. The screening data are repeated three times on three different days. Means and SEs of the means are calculated using Excel. MATLAB software is used to perform the $Z$ score calculations. The raw intensity and droplet area data for each experiment are used for the calculation of $Z$ scores. $Z$ scores are calculated by subtracting the overall average of either intensity or droplet area (within a single experiment) from the raw intensity or droplet area data for each component and dividing that result by the SD of all the measured intensities or droplet areas, according to the formula:

$$
Z \text { score }=\left(\text { intensity }_{c}-\text { mean intensity }{ }_{C 1 \ldots C n}\right) / \mathrm{SD}_{C 1 \ldots C n}
$$

where $C$ is any component on the microarray and $C 1 \ldots C n$ represent the aggregate measure of all of the components.

\section{RESULTS AND DISCUSSION}

Lipids (long-chain triglycerols-LCTs and medium-chain triglycerols-MCTs) approved by the regulatory agencies, alone or in combination, are generally first choice for developing drug carrier formulations (Marten et al., 2006; Hippalgaonkar
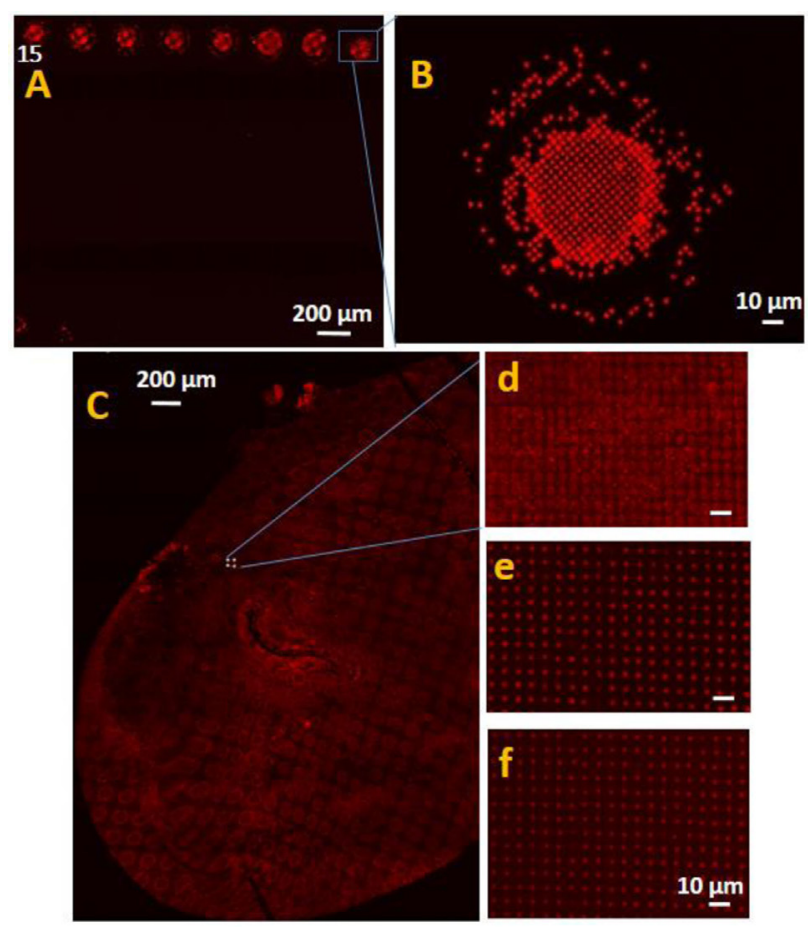

FIGURE 9 | The effect of immersion under water on liquid lipid stability of the samples stored under nitrogen atmosphere. (A) Fluorescence micrographs of castor oil/hexanoic acid combination in lipid microarray format $1 \mathrm{~h}$ after immersion under water and (B) magnified section of (A). (C) Fluorescence micrograph of a large spot of castor oil/hexanoic acid combination printed using the nanointaglio method, (D) magnified section of (C) indicated by blue square in (C); (E) fluorescence micrographs of the same spot after $1 \mathrm{~h}$ and (F) after $72 \mathrm{~h}$ immersion under water.

et al., 2010). LCTs such as soybean oil, safflower oil, sesame oil, and castor oil are approved for clinical use. Some oils (e.g., safflower, olive, sunflower, and castor) that contain more than $70 \%$ of oleic, linoleic, or ricinoleic acids make the larger spots. Our microarray includes both LCTs and MCTs and their combinations. Some oils such as linseed, safflower, and olive oils have higher fluorescence intensity, which is attributed to their autofluorescence properties (Sikorska et al., 2012). It is worth mentioning that the maximum fluorescence intensity of each spot is used in analyzing the data. Also, area values that are smaller than $3000 \mu \mathrm{m}^{2}$ have not been considered.

In the fluorescence micrograph of the palette presented in Figure 3 (Figure S1 in Supplementary Material), it is evident that not all the lipid mixtures are compatible with the pin-spotting step. Some of the components have not been pin spotted properly, as they show no fluorescence intensity. In addition, some of the samples have covered very limited area, which is almost negligible. In Figure $4 \mathrm{~B}, Z$ scores provide a relative, semiquantitative estimate of either intensity or droplet area levels and, as such, form the basis of comparison of either intensity or droplet area data among many experiments within the same array type. Thus, $Z$ scores provide a useful and intuitive method for visualizing and interpreting very large amounts of data in their natural physicochemical context. This is in contrast to normalization strategies 


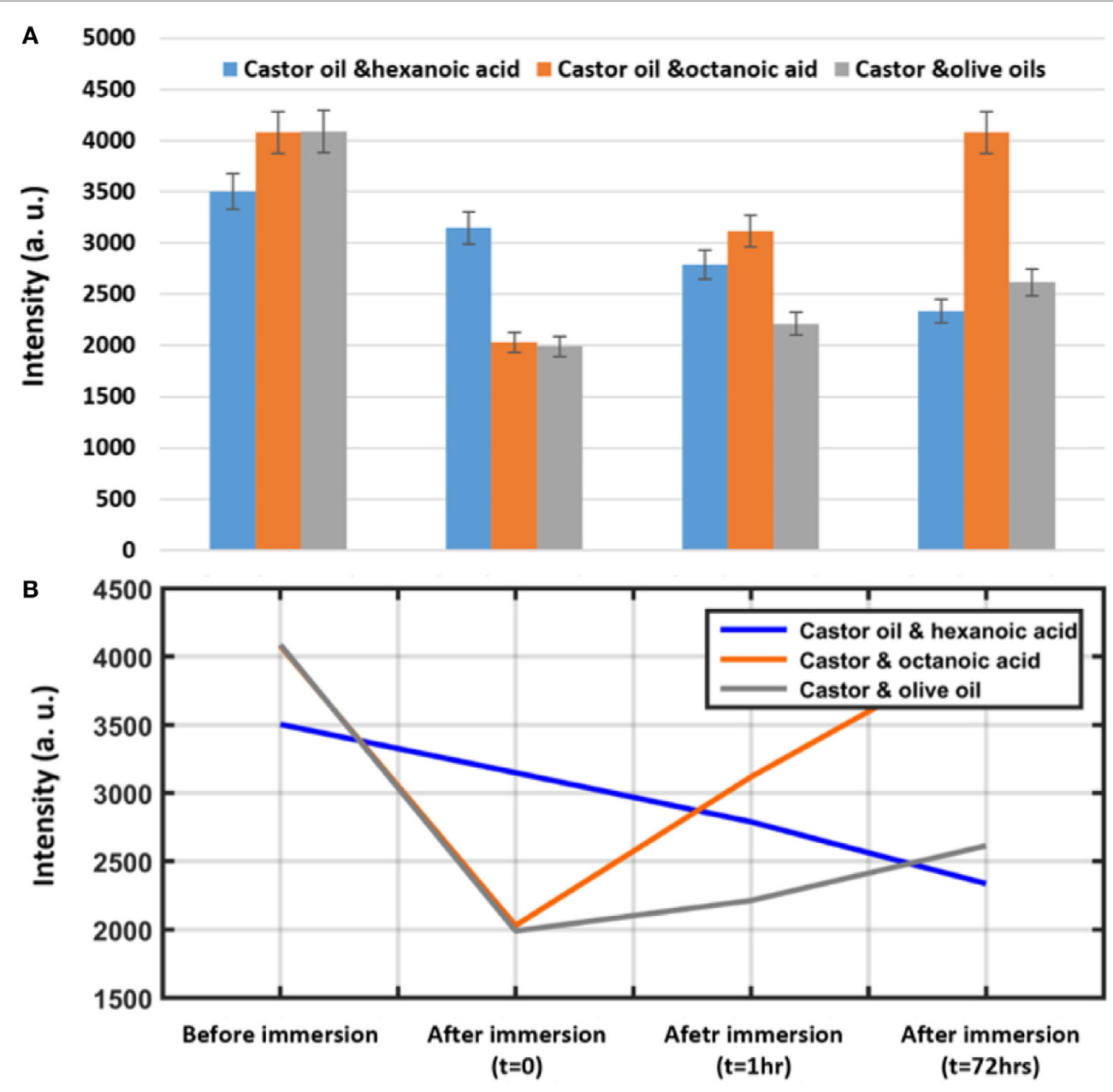

FIGURE 10 | (A) Quantification of fluorescence intensity change of a spot printed using the nanointaglio method before immersion, immediately after immersion $(t=0)$, and 1 and $72 \mathrm{~h}$ after immersion under water. Error bars represent the SEM of three different replicates. (B) Descriptive analysis of intensity versus time.

that express either intensity or droplet area data as ratios of one sample to another (either experimental or to a common reference sample). Positive and negative values in these analyses simply indicate their relationship to the normalizing sample rather than reflecting actual area or intensity levels. The very brightest dots are saturated, indicating that a sufficiently large amount of dye per dot as fluorescence intensity is related to droplet height (Nafday and Lenhert, 2011). Droplet area is likely related to both droplet volume and the contact angle of the oil on the glass surface. The viscosity of the oil and contact time of the tip may also play a role in the lipid transfer from the pin to the surface.

Castor oil, which contains monounsaturated fatty acyls, shows the most stable formulation after immersion, especially when combined with other components. Vegetable oils contain various triglycerides in different proportions; castor oil, in particular, deviates from the other oils by the high content of a monounsaturated fatty acid [ricioleic acid, 18:19 (12OH)] with a hydroxy group. For example, the free fatty acids contained in castor oil can act as a coemulsifier resulting in lower interfacial tension and more stable formulation in comparison with the other oil phases
(Mohan et al., 2012). Compared to other vegetable oils, castor oil exhibits enhanced solubilizing effects that can be ascribed to increased hydrogen bonding activities of the hydroxyl groups in ricinoleic acids.

Furthermore, it has been shown that by combining castor oil and a liquid fatty acid, at the ratio of $1: 1(w / w)$, the stability of the material under water is increased. Jumaa and Muller (1998, 1999) reported the effect of mixing castor oil with medium chain triglycerides on the viscosity of castor oil. The oil combination, at the ratio of $1: 1(\mathrm{w} / \mathrm{w})$, led to a decrease in the viscosity of castor oil and simultaneously to a decrease in the interfacial tension of the oil phase (Mohan et al., 2012). This was related to the free fatty acids contained in castor oil, which can act as a coemulsifier resulting in lower interfacial tension and, simultaneously, in a more stable formulation in comparison with the other oil phases.

In our microarray, castor oil/hexanoic acid (MCT), castor oil/ octanoic acid (MCT), and castor oil/olive oil (LCT) combinations make small patterns after pin spotting with almost uniform light intensity distribution throughout the sample, and they make good printed patterns that are reproducible. As shown in Figure 9, for 
castor oil/hexanoic acid combination, an irregular pattern of droplets is formed.

The dots are stable after immersion under water for $1 \mathrm{~h}$ in terms of the size, which demonstrates that the dots are not spreading; however, their intensity decreased during the time. As shown in Figure 10A, castor oil/octanoic acid combination shows almost complete fluorescence recovery $72 \mathrm{~h}$ after immersion under water. Intensities shown in Figure $\mathbf{1 0}$ represent the average of 30 different areas measured on three different replicate samples (10 images each). The castor/olive oil combination shows a lower fluorescence recovery compared to the castor oil/octanoic acid combination. However, the castor oil/hexanoic acid combination shows a continuous decrease in fluorescence during that time. The latter finding may suggest a mixture more prone to TRITC (and maybe drug) release over time in aqueous solutions.

Both castor oil and MCTs (hexanoic acid) are among the excipients that are being used for the manufacturing of ocular compatible lipid emulsion (Mohan et al., 2012). However, prior to the formulation of the lipid emulsions, data are needed concerning drug solubility in the oil vehicle. In addition, information is needed on compatibility of the oil vehicle with other formulation additives and with the established ocular tissue, before the dosage forms can be prepared. Our results indicate that microdroplet arrays of castor oil combinations on surfaces are suitable for screening of drugs in a scalable manner.

\section{CONCLUSION}

A screen was carried out to identify oils compatible with pin spotting and nanointaglio, followed by immersion of the microarray into water. We tested 210 lipid formulations, and a 1:1 mixture of castor oil and hexanoic acid was found to be optimal in terms of droplet size, reproducibility of printed patterns, florescence intensity, and stability under immersion. Compared to

\section{REFERENCES}

Anna, S. L., Bontoux, N., and Stone, H. A. (2003). Formation of dispersions using "flow focusing" in microchannels. Appl. Phys. Lett. 82, 364-366. doi:10.1063/1.1537519

Arrabito, G., Galati, C., Castellano, S., and Pignataro, B. (2013). Luminometric sub-nanoliter droplet-to-droplet array (LUMDA) and its application to drug screening by phase I metabolism enzymes. Lab. Chip 13, 68-72. doi:10.1039/ c2lc40948h

Bailey, S. N., Sabatini, D. M., and Stockwell, B. R. (2004). Microarrays of small molecules embedded in biodegradable polymers for use in mammalian cellbased screens. Proc. Natl. Acad. Sci. U.S.A. 101, 16144-16149. doi:10.1073/ pnas.0404425101

Cahill, D. J. (2001). Protein and antibody arrays and their medical applications. J. Immunol. Methods 250, 81-91. doi:10.1016/S0022-1759(01)00325-8

Comley, J. (2006). “Tools and technologies that facilitate automated screening” in High-Throughput Screening in Drug Discovery, ed. J. Hueser (Weinheim: WileyVCH), 37-73.

Floyd, A. G. (1999). Top ten considerations in the development of parenteral emulsions.Pharm.Sci.Technol. Today2,134-143.doi:10.1016/S1461-5347(99)00141-8

Gosalia, D. N., and Diamond, S. L. (2003). Printing chemical libraries on microarrays for fluid phase nanoliter reactions. Proc. Natl. Acad. Sci. U.S.A. 100, 8721-8726. doi:10.1073/pnas.1530261100

Heller, M. J. (2002). DNA microarray technology: devices, systems, and applications. Annu. Rev. Biomed. Eng. 4, 129-153. doi:10.1146/annurev. bioeng.4.020702.153438 phospholipid carriers (Kusi-Appiah et al., 2015), this formulation can be arrayed without the need for an additional solvent. The lipid itself can be considered the solvent for the fabrication of drug screening microarrays. These "solvent-free" lipid multilayer microarrays have potential for HTS of lipophilic compounds.

\section{AUTHOR CONTRIBUTIONS}

LG carried out the experiments and wrote the manuscript together with SL. SL conceived of the study and directed the experiments.

\section{ACKNOWLEDGMENTS}

LG would like to thank Aubrey Kusi-Appiah at the Florida State University for helpful discussions. The authors thank Jen Kennedy for proofreading.

\section{FUNDING}

This work was supported by NIH R01 GM107172.

\section{SUPPLEMENTARY MATERIAL}

The Supplementary Material for this article can be found online at http://journal.frontiersin.org/article/10.3389/fmats.2016.00055/ full\#supplementary-material.

FIGURE S1 | Fluorescence micrograph of liquid lipid-based components pin spotted on PDMS palette. Scale bar is $200 \mu \mathrm{m}$.

FIGURE S2 | Fluorescence micrograph of a liquid lipid-based microarray printed using the nanointaglio method.

FIGURE S3 | Fluorescence micrograph of the liquid lipid-based microarray $1 \mathrm{~h}$ after immersion under water, which shows the effect of immersion under water on samples stability.

Hippalgaonkar, K., Majumdar, S., and Kansara, V. (2010). Injectable lipid emulsions - advancements, opportunities and challenges. AAPS PharmSciTech 11, 1526-1540. doi:10.1208/s12249-010-9526-5

Hirtz, M., Sekula-Neuner, S., Urtizberea, A., and Fuchs, H. (2015). "Functional lipid assemblies by dip-pen nanolithography and polymer pen lithography," in Soft Matter Nanotechnology: From Structure to Function, eds X.Chen and H.Fuchs (Weinheim: Wiley-VCH), 161-185.

Hong, J., Edel, J. B., and deMello, A. J. (2009). Micro-and nanofluidic systems for high-throughput biological screening. Drug Discov. Today 14, 134-146. doi:10.1016/j.drudis.2008.10.001

Hook, A. L., Thissen, H., and Voelcker, N. H. (2006). Surface manipulation of biomolecules for cell microarray applications. Trends Biotechnol. 24, 471-477. doi:10.1016/j.tibtech.2006.08.001

Howbrook, D. N., van der Valk, A. M., O’Shaughnessy, M. C., Sarker, D. K., Baker, S. C., and Lloyd, A. W. (2003). Developments in microarray technologies. Drug Discov. Today 8, 642-651. doi:10.1016/S1359-6446(03)02773-9

Jumaa, M., and Muller, B. W. (1998). The effect of oil components and homogenization condition on the physicochemical properties and stability of parenteral fat emulsions. Int. J. Pharm. 163, 81-89. doi:10.1016/S03785173(97)00369-4

Jumaa, M., and Muller, B. W. (1999). Physicochemical properties of chitosan-lipid emulsions and their stability during the autoclaving process. Int. J. Pharm. 183, 175-184. doi:10.1016/S0378-5173(99)00086-1

Kalepu, S., and Nekkanti, V. (2015). Insoluble drug delivery strategies: review of recent advances and business prospects. Acta Pharm. Sin. B 5, 442-453. doi:10.1016/j.apsb.2015.07.003 
Kim, M., Pan, M., Gai, Y., Pang, S., Han, C., Yang, C., et al. (2015). Optofluidic ultrahigh-throughput detection of fluorescent drops. Lab. Chip 15, 1417-1423. doi:10.1039/c4lc01465k

Kusi-Appiah, A. E., Lowry, T. W., Darrow, E. M., Wilson, K. A., Chadwick, B. P., Davidson, M. W., et al. (2015). Quantitative dose-response curves from subcellular lipid multilayer microarrays. Lab. Chip 15, 3397-3404. doi:10.1039/ c5lc00478k

Kusi-Appiah, A. E., Vafai, N., Cranfill, P. J., Davidson, M. W., and Lenhert, S. (2012). Lipid multilayer microarrays for in vitro liposomal drug delivery and screening. Biomaterials 33, 4187-4194. doi:10.1016/j.biomaterials.2012.02.023

Lenhert, S., Brinkmann, F., Laue, T., Walheim, S., Vannahme, C., Klinkhammer, S., et al. (2010). Lipid multilayer gratings. Nat. Nanotechnol. 5, 275-279. doi:10.1038/nnano.2010.17

Lenhert, S., Sun, P., Wang, Y., Fuchs, H., and Mirkin, C. A. (2007). Massively parallel dip-pen nanolithography of heterogeneous supported phospholipid multilayer patterns. Small 3, 71-75. doi:10.1002/smll.200600431

Lowry, T. W., Kusi-Appiah, A., Guan, J., Van Winkle, D. H., Davidson, M. W., and Lenhert, S. (2014). Materials Integration by nanointaglio. Adv. Mater. Interfaces 1, 1300121-1300125. doi:10.1002/admi.201300127

Ma, H., and Horiuchi, K. Y. (2006). Chemical microarray: a new tool for drug screening and discovery. Drug Discov. Today 11, 661-668. doi:10.1016/ j.drudis.2006.05.002

Marten, B., Pfeuffer, M., and Schrezenmeir, J. (2006). Medium-chain triglycerides. Int. Dairy J. 16, 1374-1382. doi:10.1016/j.idairyj.2006.06.015

Mohan, K., Pravin, S., and Atul, B. (2012). Ophthalmic microemulsion: a comprehensive review. Int. J. Pharma. Bio. Sci. 3, 1-13.

Mugherli, L., Burchak, O. N., Balakireva, L. A., Thomas, A., Chatelain, F., and Balakirev, M. Y. (2009). In situ assembly and screening of enzyme inhibitors with surface-tension microarrays. Angew. Chem. Int. Ed. 121, 7775-7780. doi:10.1002/ange.200901139

Nafday, O. A., and Lenhert, S. (2011). High-throughput optical quality control of lipid multilayers fabricated by dip-pen nanolithography. Nanotechnology 22, 225301. doi:10.1088/0957-4484/22/22/225301

Nafday, O. A., Lowry, T. W., and Lenhert, S. (2012). Multifunctional lipid multilayer stamping. Small 8, 1021-1028. doi:10.1002/smll.201102096
Pirrung, M. C. (2002). How to make a DNA chip. Angew. Chem. Int. Ed. 41, 1276-1289. doi:10.1002/1521-3773(20020415)41:8<1276::AID-ANIE1276> 3.0.CO;2-2

Popova, A. A., Demir, K., Hartanto, T. G., Schmitta, E., and Levkin, P. A. (2016). Droplet-microarray on superhydrophobic-superhydrophilic patterns for high-throughput live cell screenings. RSC Adv. 6, 38263-38276. doi:10.1039/ C6RA06011K

Rohrer, H. (1996). The nanoworld: chances and challenges. Microelectron. Eng. 32, 5-14. doi:10.1016/0167-9317(95)00173-5

Sikorska, E., Khmelinskii, I., and Sikorski, M. (2012). "Analysis of olive oils by fluorescence spectroscopy: methods and applications," in Olive Oil - Constituents, Quality, Health Properties and Bioconversions, ed. D. Boskou (InTech). Available at: http://www.intechopen.com/books/olive-oil-constituents-quality-healthproperties-and-bioconversions/analysis-of-olive-oils-by-fluorescencespectroscopy-methods-and-applications

Sun, Y., Chen, X., Zhou, X., Zhu, J., and Yu, Y. (2015). Droplet-in-oil array for picoliter-scale analysis based on sequential inkjet printing. Lab. Chip 15, 2429-2436. doi:10.1039/c5lc00356c

Vafai, N., Lowry, T. W., Wilson, K. A., Davidson, M. W., and Lenhert, S. (2015). Evaporative edge lithography of a liposomal drug microarray for cell migration assays. Nanofabrication 2, 32-42. doi:10.1515/nanofab-2015-0004

Wu, J., Wheeldon, I., Guo, Y., Lu, T., Du, Y., Wang, B., et al. (2011). A sandwiched microarray platform for benchtop cell-based high throughput screening. Biomaterials 32, 841-848. doi:10.1016/j.biomaterials.2010.09.026

Conflict of Interest Statement: The authors declare that the research was conducted in the absence of any commercial or financial relationships that could be construed as a potential conflict of interest.

Copyright (C) 2016 Ghazanfari and Lenhert. This is an open-access article distributed under the terms of the Creative Commons Attribution License (CC BY). The use, distribution or reproduction in other forums is permitted, provided the original author(s) or licensor are credited and that the original publication in this journal is cited, in accordance with accepted academic practice. No use, distribution or reproduction is permitted which does not comply with these terms. 